\title{
Erratum: Nonlinear Dirac equation solitary waves in external fields [Phys. Rev. E 86, 046602 (2012)]
}

\author{
Franz G. Mertens, Niurka R. Quintero, Fred Cooper, Avinash Khare, and Avadh Saxena \\ (Received 5 February 2016; published 10 May 2016)
}

DOI: 10.1103/PhysRevE.93.059903

In Sec. IV of our original paper, we assumed a particular conservation law Eq. (4.6), which was true in the absence of external potentials, to derive some particular potentials for which we obtained solutions to the nonlinear Dirac equation (NLDE). Because the conservation law of Eq. (4.6) for the component $T^{11}$ of the energy-momentum tensor is not true in the presence of these external potentials, the solutions we found do not satisfy the NLDEs in the presence of these potentials. Thus all the equations from Eq. (4.6) through Eq. (4.44) are not correct, since the exact solutions that followed in that section presumed Eq. (4.6) was true. Also Eqs. (A3)-(A5) are a restatement of Eq. (4.6) and also are not correct. These latter equations are also not used in Sec. V and beyond. The rest of our original paper (starting with Sec. V) was not concerned with exact solutions, rather it was concerned with how the exact solitary-wave solutions to the NLDE in the absence of an external potential responded to being in the presence of various external potentials.

In this Erratum, we correct this mistake and show how to directly find exact solutions of the NLDE in a particular class of external potentials. That is, we show how to directly solve the equations for the two components of the NLDE in $1+1$ dimension with scalar-scalar self-interaction $\frac{g^{2}}{\kappa+1}(\bar{\Psi} \Psi)^{\kappa}$ in the presence of an external electromagnetic potential in the axial gauge $e A^{0}(x)=V(x), A^{1}(x)=0$ without resorting to the conservation law of Eq. (4.6).

Writing the two components of the bound-state solution of the NLDE as $\Psi=e^{-i \omega t}\{R \cos \theta, i R \sin \theta\}$ and assuming that $V(x)$ depends on $x$ only through its dependence on $R^{2}=y$, we are able to find exact solutions of the NLDE for arbitrary $\kappa$ in these potentials.

We start with the NLDE in the presence of an external electromagnetic potential:

$$
\left(i \gamma^{\mu} \partial_{\mu}-m\right) \Psi-e \gamma^{\mu} A_{\mu} \Psi+g^{2}(\bar{\Psi} \Psi)^{\kappa} \Psi=0 .
$$

Using the freedom of gauge invariance, one can choose the axial gauge $A^{1}=0, e A_{0}=V(x)$. One can also rescale the fields so that we can set the coupling constant $g=1$. In the axial gauge, the NLDE becomes

$$
i \gamma^{\mu} \partial_{\mu} \Psi-m \Psi+(\bar{\Psi} \Psi)^{\kappa} \Psi-\gamma^{0} V(x) \Psi=0 .
$$

Going into the rest frame and choosing $\Psi(x, t)=e^{-i \omega t} \psi(x)$, and for $\psi(x)$ the representation

$$
\psi(x)=\left(\begin{array}{c}
A(x) \\
i B(x)
\end{array}\right)=R(x)\left(\begin{array}{c}
\cos \theta \\
i \sin \theta
\end{array}\right),
$$

we find that the NLDE becomes

$$
\begin{aligned}
& \partial_{x} A+(m+\omega) B-g^{2}\left[A^{2}-B^{2}\right]^{\kappa} B-V(x) B=0, \\
& \partial_{x} B+(m-\omega) A-g^{2}\left[A^{2}-B^{2}\right]^{\kappa} A+V(x) A=0 .
\end{aligned}
$$

These two equations can also be written if we let $y=R^{2}(x)$ as

$$
\frac{d y}{d x}=2 y^{\kappa+1}(\cos 2 \theta)^{\kappa} \sin 2 \theta-2 y m \sin 2 \theta
$$

and

$$
\frac{d \theta}{d x}=y^{\kappa} \cos ^{\kappa+1} 2 \theta-m \cos 2 \theta+[\omega-V(x)] .
$$

We can now follow the approach that Chang, Ellis, and Lee [1] used in obtaining exact solutions when $V=0$. Dividing Eq. (5) by Eq. (6) and assuming that $V$ is just a function of $y=R^{2}$ and furthermore setting $V[y]=d f[y] / d y$, we obtain

$$
\frac{d}{d \theta}\left[\frac{y^{\kappa+1}}{\kappa+1} \cos ^{\kappa+1} 2 \theta+[\omega-m \cos 2 \theta] y-f[y]\right]=0 .
$$

Integrating with respect to $\theta$ and assuming that we have a no-node solution going to zero at large $|x|$ so that the constant of integration is zero, we obtain

$$
y^{\kappa} \cos ^{\kappa+1} 2 \theta=-(\kappa+1)[\omega-m \cos 2 \theta-f(y) / y] .
$$

Substituting this result into Eq. (6), one obtains the equation

$$
\frac{d \theta}{d x}=-\kappa(\omega-m \cos 2 \theta)-\left(\frac{d f}{d y}-(\kappa+1) \frac{f}{y}\right) .
$$


We notice that if we choose $f$ to be a solution of

$$
\frac{d f}{d y}-(\kappa+1) \frac{f}{y}=0
$$

i.e.,

$$
f=v_{0} \frac{y^{\kappa+1}}{\kappa+1},
$$

so that

$$
V[y]=v_{0} y^{\kappa},
$$

we obtain the same equation for $\theta$ as when $V[y]=0$. Namely,

$$
\frac{d \theta}{d x}=-\kappa(\omega-m \cos 2 \theta)
$$

whose solution is

$$
\theta(x)=\tan ^{-1}\left[\alpha \tanh \beta_{\kappa}(x)\right]
$$

where $\alpha=\sqrt{\frac{m-\omega}{m+\omega}}$ and $\beta_{\kappa}=\kappa \sqrt{m^{2}-\omega^{2}}$.

When $\kappa=1$, this solution was obtained by different means by Nogami and Toyama [2]. Now we can solve for $y=R^{2}$ by using Eq. (8) to obtain

$$
R^{2}=\left[\frac{(\kappa+1)(m \cos 2 \theta-\omega)}{\cos ^{\kappa+1} 2 \theta-v_{0}}\right]^{1 / \kappa}
$$

This can also be written using the fact that

$$
\cos 2 \theta=\frac{m+\omega \cosh 2 \beta_{\kappa} x}{\omega+m \cosh 2 \beta_{\kappa} x}
$$

as

$$
R^{2}=\left(\frac{(\kappa+1)\left(m^{2}-\omega^{2}\right)}{\left[m \cosh \left(2 \beta_{\kappa} x\right)+\omega\right]\left[\left(\frac{m+\omega \cosh \left(2 \beta_{\kappa} x\right)}{m \cosh \left(2 \beta_{\kappa} x\right)+\omega}\right)^{\kappa+1}-v_{0}\right]}\right)^{\frac{1}{\kappa}} .
$$

This reduces to our previous result when $v_{0} \rightarrow 0$, i.e.

$$
R^{2}=\left(\frac{m \cosh \left(2 \beta_{\kappa} x\right)+\omega}{m+\omega \cosh \left(2 \beta_{\kappa} x\right)}\right)\left[\frac{(\kappa+1) \beta_{\kappa}^{2}}{\kappa^{2}\left(m+\omega \cosh 2 \beta_{\kappa} x\right)}\right]^{1 / \kappa} .
$$

Now $R^{2}$ has to be positive and vanish when $|x| \rightarrow \infty$, which means that for $\kappa=1, v_{0}<\omega^{2} / m^{2}$. When $v_{0}<0$ one has an attractive potential, and this type of solution always exists. As $v_{0}$ approaches $\omega^{2} / \mathrm{m}^{2}, R^{2}$ can start to become double-humped. We show some results for $\kappa=1$ in the figures. For example, when $\omega=7 / 10$ and we go from $v_{0}=0.4$ to 0.48 , the shape of $R^{2}$ shifts from single-humped to double-humped, as seen in Fig. 1 . For $v_{0} \geqslant 0.49$, there are no solutions that vanish when $|x| \rightarrow \infty$. Choosing $v_{0}=-1$ and $\omega=1 / 2$, we obtain instead the results of Fig. 2 for the bound-state solution.

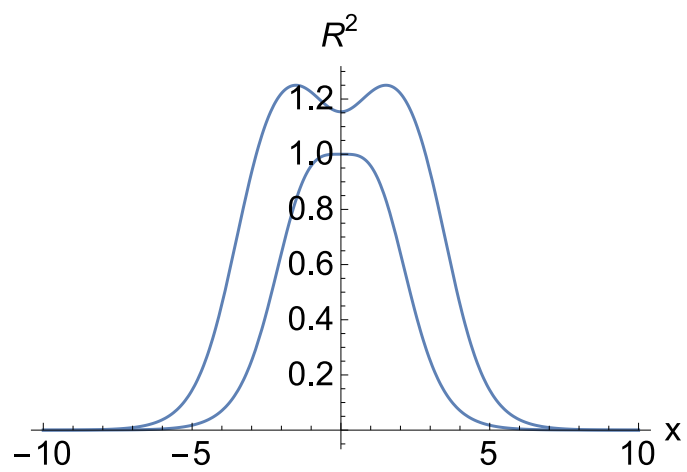

FIG. 1. $R^{2}$ vs $x$ when $\omega=7 / 10, v_{0}=0.4$ (lower curve), and $v_{0}=0.48$ (upper curve) for the bound-state solution in the external potential $V=v_{0} R^{2}$ 


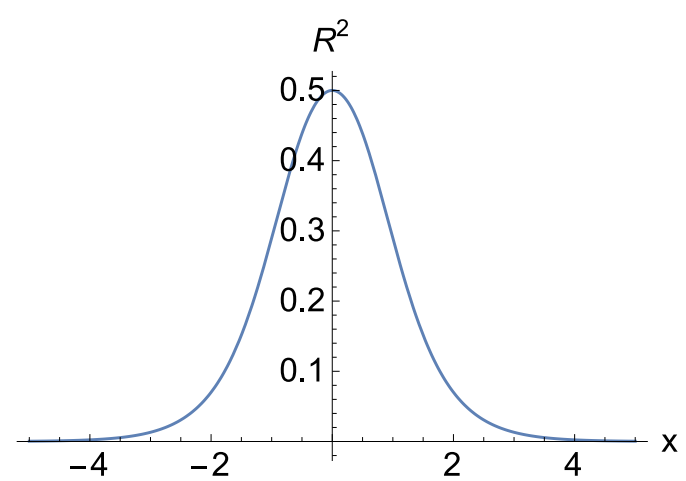

FIG. 2. $R^{2}$ vs $x$ when $\omega=1 / 2$ and $v_{0}=-1$ for the bound-state solution in the external potential $V=-R^{2}$.

This work was supported in part by the U.S. Department of Energy. F.G.M. acknowledges the hospitality of the Mathematical Institute of the University of Seville (IMUS) and of the Theoretical Division and Center for Nonlinear Studies at Los Alamos National Laboratory, and the financial support by the Plan Propio of the University of Seville and by Junta de Andalucia. N.R.Q. acknowledges financial support from the Humboldt Foundation through Research Fellowship for Experienced Researchers SPA 1146358 STP (Germany) and by the MINECO through FIS2014-54497-P (Spain).

[1] S.-J. Chang, S. D. Ellis, and B. W. Lee, Phys. Rev. D 11, 3572 (1975).

[2] Y. Nogami and F. M. Toyama, Phys. Rev. A 45, 5258 (1992). 